\title{
INFLUENCE OF FLUORINATION ON UV SPECTRA OF POLYURETHANE STRUCTURAL FRAGMENTS
}

\author{
M. A. Ksenofontova V. V. Ponarjadov, ${ }^{\text {b D. S. Umreiko, }}{ }^{a}$ \\ M. B. Shundalau, ${ }^{b^{*}}$ and E. J. Bobkova ${ }^{a}$
}

UDC 539.19

Results of TDDFT calculations of the characteristics for excited singlet states of fluorinated urethanes containing from one to six fluorine atoms were presented. The influence of the number and location of the fluorine atoms in the urethane structure on the formation of its UV absorption spectrum was analyzed. It was established that fluorination of a phenyl group in the ortho-positions led to steric hindrance in the urethane structure and to rotation of the urethane group by a significant angle (of about $20^{\circ}$ ). The rotation angle of the urethane group could reach $60^{\circ}$ and more with additional fluorination of the amine. Such changes in the urethane structure were accompanied by characteristic significant bathochromic shifts of the long-wavelength absorption bands.

Keywords: ab initio calculation, time-dependent density functional theory, UV spectrum, urethanes, fluorination.

Introduction. The cellular structure of polyurethane foams, i.e., gas-filled heterochain polymers containing both hydrophobic and hydrophilic functional structural elements, enables effective sorption filters to be fabricated from them [1]. The membrane-like structure of the cell and pore walls provides access to the sorbing substance within the sorbent and is responsible for its high absorbance and retention. The sorbing substance is involved in both adsorption and absorption $[1,2]$.

Additional possibilities for expanding the range of applications of polyurethane foams exist despite the high operational parameters of sorbents based on them. Thus, chemical modification of solid surfaces is a common method for a tailored change of their properties [3]. Modified sorbents exhibit several advantages over their ordinary analogs because of the combination of properties of the modifiers and the surface characteristics of the solid matrix. An effective method for modification of polymer surfaces is direct fluorination. This can improve certain operational characteristics of polymeric materials including their sorbing properties [4]. Fluorination of polymers replaces a $\mathrm{H}$ atom by fluorine and binds the latter to $\mathrm{C}=\mathrm{C}$ and $\mathrm{C}-\mathrm{N}$ bonds (with $\mathrm{N}$ substitution). Thus, determination of the chemical composition and structure of the modified polymer is often ambiguous.

We have previously analyzed changes of electronic absorption spectra caused [5] by various amounts of functional groups in the structure of a urethane chain using quantum-chemical time-dependent density functional theory (TDDFT) [6] to model the electronic structure of isocyanates and carbamates. It was shown that the position of the absorption band in the electronic spectrum was determined mainly by the number of structural elements (phenyl and urethane groups) included in the carbamate. Time-dependent DFT methods are currently used successfully to solve spectral analysis problems [7-11] because a typical quantum-chemical calculation carried out in terms of TDDFT using, e.g., a B3LYP hybrid functional, gave energies of excited states that deviated from the experimental values by $0.4 \mathrm{eV}[6]$.

Herein we present results of quantum-chemical TDDFT calculations of the characteristics of excited electronic states of two urethane representatives, i.e., methylphenylcarbamate (MPC) and 4,4'-diphenylmethane-bis(methyl)carbamate (DPMC), several $\mathrm{H}$ atoms of which were substituted by $\mathrm{F}$ atoms. The structures of various F-substituted carbamates and the influence of the conformations of such compounds on the electronic absorption spectra have not previously been studied using systematic quantum-chemical calculations. These urethanes included in their composition functional groups belonging to a typical polyurethane (urethane, phenyl, methyl, and methylene) and acted as structural models of the polymer fragments.

\footnotetext{
*To whom correspondence should be addressed.
}

${ }^{a}$ A. N. Sevchenko Institute of Applied Physical Problems, Belarusian State University, Minsk; e-mail: lab_dozator@ mail.ru; ${ }^{b}$ Belarusian State University, 4 Nezavisimosti Ave., Minsk, 220030, Belarus; e-mail: shundalov@bsu.by. Translated from Zhurnal Prikladnoi Spektroskopii, Vol. 80, No. 3, pp. 330-336, May-June, 2013. Original article submitted June 29, 2012. 
Fluorination of the organic molecules could cause substantial changes in their steric structure because the atomic radius of $\mathrm{F}$ is significantly greater than that of $\mathrm{H}$. Therefore, the electronic absorption spectra demonstrated a definite sensitivity to structural changes of the molecular systems. The shape of such spectra contained important information about the number and location of $\mathrm{F}$ atoms in the molecular structure.

Calculations. Spectral characteristics of the examined compounds were calculated using the applied quantumchemical program GAMESS-US [12, 13]. The results were visualized using the MacMolPIt [14] and ORTEP [15] programs. The presence of carbonyl and phenyl groups in MPC allowed it to be classified as a molecular system for which intramolecular charge transfer (ICT) could be characteristic. According to the classification of Freimanis [16], MPC should be considered a donor-acceptor system with a one-membered bridge where the phenyl group acts as the donor (D); the carbonyl, the acceptor (A); and the $\mathrm{N}$ heteroatom, the bridge. Freimanis noted [16] that spectra of compounds with a heteroatom (in particular, a $\mathrm{N}$ atom) by far do not always exhibit signs of ICT, in contrast with DA systems (in which the bridge is a C atom with $s p^{3}$ hybridization) that demonstrate, as a rule, clearly distinguished ICT bands. One of the possible reasons for this relates to the donor properties of the amine itself. Thus, a band near $250 \mathrm{~nm}$ in the absorption spectrum of aniline [17] corresponds to ICT from the amine to the phenyl.

It is well known [18-21] that methods for calculating electronic spectra that are based on a local density approximation (even taking into account gradient corrections) in terms of TDDFT do not always reproduce successfully the spectral and energy characteristics of the excited electronic states of molecular systems with intra- and intermolecular charge transfer. In this instance, one of the reasons for the errors in the calculations is the diffuseness of the ICT process (the sources of the errors has been analyzed in detail [19]). It is noteworthy that this fundamental inadequacy of the local density approximation does not appear for all molecular systems with ICT. Such a situation in general is characteristic of DFT calculations [22]. Therefore, single procedures or guidelines enabling a method (and also functional, basis, etc.) to be selected for an adequate calculation of a particular molecular system do not exist.

One method of accounting for diffuseness in ICT processes is the long-range correction (LRC) method [23, 18]. It is based on separation of a two-electron coupling operator into short-range and long-range parts. It is noteworthy that the LRC method is obviously aimed at correcting the characteristics of separate excited states (namely, with ICT) and not the whole system of levels.

The TDDFT/B3LYP/cc-pVDZ theory level has been used to calculate the spectral and energy characteristics of excited electronic states [24-27]. Because the B3LYP functional does not allow LRC corrections, BLYP [25, 26] and BLYP + LRC functionals were also used. Compounds that a) had functional groups typical of MPC in their structure; b) were examples of molecules both without and with ICT; and c) were sufficiently studied experimentally were examined initially in order to determine the adequacy of the various types of used approximations (reproduction of energies of excited states, oscillator strengths, ordering of energy levels).

Aniline and its 4-Cl-, 5-Cl-, and 5-F-substituted analogs in addition to benzene and benzaldehyde were used as such test subjects. Aniline and benzaldehyde were examples of compounds with ICT. The phenyl group acted as the acceptor in the former; as the donor, in the latter. Systems of singlet excited states of aniline and its halo-substituted derivatives were studied previously [17]. Experimental data for singlet and triplet states of benzene and benzaldehyde were reviewed [28, 29].

The following energy values were obtained for the lower electronic states of benzene in the B3LYP/cc-pVDZ approximation: $T_{1}=3.78, T_{2}=4.73, T_{3}=5.12, S_{1}=5.46, S_{2}=6.20, T_{4}=7.24, S_{3}=7.24 \mathrm{eV}$; in the BLYP/cc-pVDZ approximation: $T_{1}=4.04, T_{2}=4.66, T_{3}=4.98, S_{1}=5.27, S_{2}=6.06, T_{4}=7.81, S_{3}=6.93 \mathrm{eV}$; in the BLYP + LRC/cc-pVDZ approximation: $T_{1}=3.67, T_{2}=4.89, T_{3}=5.11, S_{1}=5.50, S_{2}=6.40, T_{4}=7.57 \mathrm{eV}$. The corresponding experimental energies were: $T_{1}=3.9, T_{2}=4.7, S_{1}=4.78-5.20$ (taking into account vibrational structure), $T_{3}=5.6, S_{2}=6.20-6.53, T_{4}=6.55, S_{3}$ $=6.95 \mathrm{eV}$ [28]. It can be seen that calculations using B3LYP and BLYP functionals in general reproduced successfully the experimental data with the exception of the ordering of the $T_{3}$ and $S_{1}$ states relative to the experimental values. A calculation using an LRC correction gave as expected poor results because ICT is uncharacteristic of benzene.

For benzaldehyde (the $S_{0} \rightarrow S_{2}$ transition is accompanied by ICT from the phenyl to the carbonyl) in the B3LYP/ cc-pVDZ approximation, the following energies were obtained (for triplet states energies of only $T_{1}$ and $T_{2}$ are given; here and later normalized oscillator strengths are shown in parentheses): $T_{1}=3.09(0), T_{2}=3.29(0), S_{1}=3.65(0), S_{2}=4.76(0.08)$, $S_{3}=5.28(1.0), S_{4}=5.54(0), S_{5}=6.34(0.60) \mathrm{eV}$; in the BLYP/cc-pVDZ approximation: $T_{1}=2.80(0), T_{2}=3.41(0), S_{1}=$ 3.26(0), $S_{2}=4.30(0), S_{3}=4.36(0.07), S_{4}=4.98(1.0), S_{5}=5.66(0.01) \mathrm{eV}$; in the BLYP + LRC/cc-pVDZ approximation: $T_{1}$ $=3.17(0), T_{2}=3.27(0), S_{1}=3.79(0), S_{2}=5.10(0.05), S_{3}=5.60(0.81), S_{4}=6.80(1.0), S_{5}=6.92(0) \mathrm{eV}$. The corresponding experimental energies were $T_{1}=3.12, T_{2}=3.23, S_{1}=3.34, S_{2}=4.51, S_{3}=5.34$, no data for $S_{4}$, and $S_{5}=6.36 \mathrm{eV}$ [29]. Thus, 
the calculation using the hybrid functional B3LYP agreed best with the experimental data because the functional BLYP gave zero oscillator strength for the $S_{0} \rightarrow S_{2}$ transition with ICT and reduced the energy of the $S_{3}$ state by almost $1 \mathrm{eV}$. Calculations with functionals BLYP and BLYP + LRC produced an incorrect ordering of the $S_{4}$ and $S_{5}$ states.

Functional BLYP gave the least elevated energies of the three versions of calculations for aniline (the $S_{0} \rightarrow S_{2}$ transition is accompanied by ICT from the amine to phenyl): in the B3LYP/cc-pVDZ approximation $S_{1}=4.81(0.29), S_{2}=$ 5.63(1.0), $S_{3}=5.99(0.06) \mathrm{eV}$; in the BLYP/cc-pVDZ approximation $S_{1}=4.45(0.31), S_{2}=5.31(1.0), S_{3}=5.57(0.49) \mathrm{eV}$; in the BLYP + LRC/cc-pVDZ approximation $S_{1}=4.98(0.26), S_{2}=5.86(1.0), S_{3}=6.43(0.02) \mathrm{eV}$. The experimental energies were $S_{1}=4.23(0.17)$ [17], $S_{2}=4.60(0)$ [30], and $S_{3}=4.96(1.0) \mathrm{eV}$ [17]. All three versions of the calculations gave an incorrect ordering of states. The calculation with functional BLYP increased the oscillator strength for the transition with zero intensity. For 4-Cl-aniline \{B3LYP/cc-pVDZ: $S_{1}=4.46(1.0), S_{2}=4.64(0) \mathrm{eV}$; BLYP/cc-pVDZ: $S_{1}=4.01(0), S_{2}=4.12(1.0) \mathrm{eV}$; BLYP + LRC/cc-pVDZ: $S_{1}=4.67(1.0), S_{2}=5.44(0) \mathrm{eV}$; experimental energies [17] were $\left.S_{1}=4.03(0.17), S_{2}=4.90(1.0)\right\}$, calculations using B3LYP and BLYP + LRC gave zero oscillator strength for the transition with ITC whereas the calculation using BLYP decreased significantly the energy of this state. For 5-Cl-aniline \{B3LYP/cc-pVDZ: $S_{1}=4.13(0), S_{2}=4.27(1.0), S_{3}=4.80(0)$ eV; BLYP/cc-pVDZ: $S_{1}=3.50(0), S_{2}=3.91(1.0), S_{3}=4.19(0) \mathrm{eV}$; BLYP + LRC/cc-pVDZ: $S_{1}=4.49(1.0), S_{2}=4.94(0), S_{3}$ $=5.36(0.53) \mathrm{eV}$; the experimental energies [17] were $\left.S_{1}=3.80(0.13), S_{2}=4.81(0.51), S_{3}=5.34(1.0) \mathrm{eV}\right\}$, all calculations did not agree satisfactorily with the experimental values. The situation was analogous for 5-F-aniline \{B3LYP/cc-pVDZ: $S_{1}=$ 4.93(1.0), $S_{2}=5.08(0) \mathrm{eV}$; BLYP/cc-pVDZ: $S_{1}=4.45(0), S_{2}=4.54(1.0) \mathrm{eV} ; \mathrm{BLYP}+\mathrm{LRC} / \mathrm{cc}-\mathrm{pVDZ}: S_{1}=5.06(1.0), S_{2}=$ $5.50(0) \mathrm{eV}$; experimental energies [17] were $\left.S_{1}=4.41(0.31), S_{2}=5.23(1.0) \mathrm{eV}\right\}$.

Thus, use of the TDDFT method (even for calculating the LRC) to model spectral and energy characteristics of excited states of DA-systems with ICT to a phenyl turned out to be unjustified. This calculation method did in general give acceptable results for DA-systems with ICT from a phenyl. In this instance, calculation of the LRC did not significantly improve them.

Results and Discussion. Equilibrium configurations were found for F-substituted MPC (Fig. 1a and b) upon replacing one $\mathrm{H}$ atom of the $\mathrm{NH}$ group (1-substitution), the $\mathrm{C}_{6} \mathrm{H}_{5}$ group (2-, 3-, 4-, 5-, and 6-substitution), and the $\mathrm{CH}_{3}$ group (7-substitution); two (1,2-, 1,3-, 1,4-, 1,5-, 1,6-, 1,7-, 2,3-, and 2,6-substitution); three (1,2,6- and 7,8,9-substitution); four (2,3,5,6-substitution); and six $\mathrm{H}$ atoms (1,2,3,4,5,6-substitution). The fact that the proposed stable configurations of the substituted MPC molecules fell on stable points of the potential energy surface was confirmed by calculating the Hessian. Three $\mathrm{H}$ atoms in the 1-, 2-, and 6-positions of DPMC could be substituted by F only in one part of the molecule (Fig. 1c). Energy characteristics (energies of states and the corresponding oscillator strengths) of 20 excited singlet electronic states were calculated for each of the configurations of F-substituted MPC; for DPMC, 30. Figure 2 shows model spectra (the positions of the longest wavelength absorption band maxima are shown).

Let us analyze firstly electronic absorption spectra of monofluorinated MPC (Fig. 2, spectra 2-8). The structure of the MPC UV absorption spectrum at excitation energies up to $8.5 \mathrm{eV}$ was in general retained upon replacing only one $\mathrm{H}$ atom by F. The longest wavelength band of weak intensity (analog of forbidden transition ${ }^{1} A_{1 g} \rightarrow{ }^{1} B_{2 u}$ in the benzene spectrum with $\lambda=256 \mathrm{~nm}$ ) was due to a $\pi \pi^{*}$-type transition. The pair of orbitals HOMO $\rightarrow$ LUMO +1 (HOMO is the highest occupied; LUMO, the lowest unoccupied molecular orbital) contributed most to this transition with less significant contributions of HOMO $-1 \rightarrow$ LUMO and HOMO $\rightarrow$ LUMO orbital pairs. As a rule, this band upon monosubstitution experienced a very weak $(\sim 1.0-1.5 \mathrm{~nm})$ hypsochromic shift relative to its position in the spectrum of MPC (248 nm, $5.00 \mathrm{eV})$. The exceptions were 4- and 6-substituted MPC, in the spectra of which the aforementioned band shifted to longer and shorter wavelength by 10 and $2.5 \mathrm{~nm}$, respectively. The bathochromic shift of 4-substituted MPC (spectrum 5) was due to noticeable redistribution of ring electron density as a result of the para-substitution; for 6-substituted (spectrum 7), the hypsochromic shift was due to a change of molecular structure. Steric hindrance of the $\mathrm{F}$ and carbonyl $\mathrm{O}$ atoms led to a deviation of the urethane group from the ring plane by $\sim 57^{\circ}$ (here and later, the deviation angle of urethane from the ring plane means the dihedral angle between the ring plane and the plane passing through the $\mathrm{NC}=\mathrm{O}$ atoms). It is noteworthy that such a structural change could have an artificial nature for monosubstituted MPC because 2- and 6-substituted MPC were rotational isomers relative to the $\mathrm{CN}$ bond. Because the energy of the 2-substituted isomer was $37 \mathrm{~kJ} / \mathrm{mol}$ less than that of the 6 -substituted isomer, the latter was an unstable isomer. Formation of an intramolecular H-bond of the CF...HN type was possible in the structure of 2-substituted MPC. This could act as an additional stabilizing factor of the isomer.

The band near $227 \mathrm{~nm}(5.46 \mathrm{eV})$ in the spectrum of MPC was due to a $\pi \pi^{*}$-type transition. It also retained its position and relative intensity in spectra of monofluorinated MPC with the exception of the obvious case of 1-substitution (spectrum 2), where the band at $230 \mathrm{~nm}(5.40 \mathrm{eV})$ had a long-wavelength satellite at $234 \mathrm{~nm}(5.30 \mathrm{eV})$. The pair of orbitals 

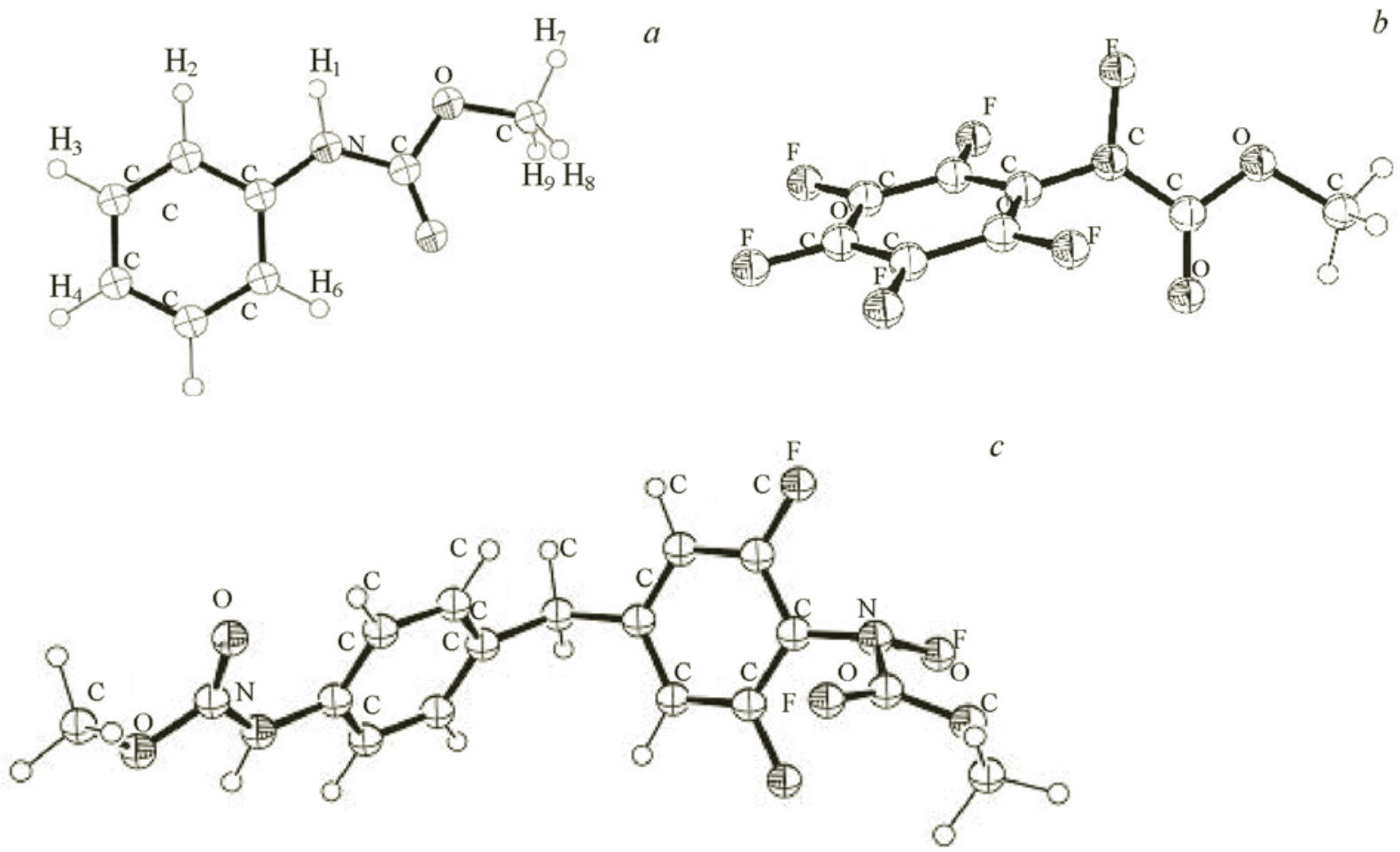

Fig. 1. Equilibrium configurations of methylphenylcarbamate $(a)$ with numbering of $\mathrm{H}$ atoms; 1,2,3,4,5,6-fluoro-substituted MPC (b); and 1,2,6-fluoro-substituted DPMC (c).

$\mathrm{HOMO} \rightarrow$ LUMO contributed most to this transition with the pairs of orbitals HOMO $-1 \rightarrow$ LUMO +1 and HOMO $\rightarrow$ LUMO +1 contributing less significantly. It should be emphasized that assigning absorption bands to certain types of transitions was somewhat arbitrary because up to 10 pairs of molecular orbitals contributed to several of the examined transitions. For example, this applied fully to a group of strong bands in the range $6.45-6.70 \mathrm{eV}$.

Fluorosubstitution in other positions (3-, 5-, and 7-) produced less significant spectral changes. Because 3- and 5 -substituted MPC were rotational isomers, their spectra were practically identical (spectra 4 and 6). The aforementioned trends were also observed in UV spectra of disubstituted MPC derivatives (spectra 9-16). For 1,2- and 1,6-substituted derivatives (which were rotational isomers with the energy of the 1,6-substituted derivative greater by $18 \mathrm{~kJ} / \mathrm{mol}$ than that of the 1,2-substituted isomer) and the 2,6-substituted MPC, steric hindrance between the two F atoms (in the first case) or F and $\mathrm{O}$ (in the second and third cases) caused the urethane group to rotate relative to the ring plane by 57,15 , and $27^{\circ}$, respectively.

Structural changes upon 1,2- and 1,6-substitution led to considerable restructuring of the UV spectra (spectra 1, 9, and 13). The band at $227 \mathrm{~nm}$ in the spectrum of MPC underwent a significant bathochromic shift (by 30 and $26 \mathrm{~nm}$, respectively), as a result of which it occurred at longer wavelength. This band for the 2,6-substituted derivative (spectrum 16) was also shifted (by $3 \mathrm{~nm}$ ) to longer wavelength. The structures of spectra with disubstitution in other positions correlated in general with the corresponding data for the monosubstituted models. The appearance in spectra of the 1,2- and 1,6-substituted derivatives (spectra 9 and 13) of weak absorption bands in the range 5.7-6.4 eV (217-194 nm) and also in spectra of the other two disubstituted rotational isomers (1,3- and 1,5-substituted, spectra 10 and 12) of a band at $193 \mathrm{~nm}(6.42 \mathrm{eV})$ was probably due to a change of the local symmetry of the phenyl electron density distribution as a result of the asymmetric F-substitution. The spectrum of 1,4-substituted MPC (para-substitution in the ring) did not show an absorption band in this region (spectrum 11).

Model spectra of 1,2,6-substituted (spectrum 17) and 1,2,3,4,5,6-substituted MPC (spectrum 21) confirmed the aforementioned trends because the urethane group in the first instance was rotated by $63^{\circ}$ relative to the ring plane; in the second, by $73^{\circ}$ (Fig. 1b). In this respect, the spectrum of 1,2,3,4,5,6-substituted MPC was most characteristic. The bands at 227 and $248 \mathrm{~nm}$ in the spectrum of MPC were shifted to long wavelength by 26 and $14 \mathrm{~nm}$, respectively. New weak absorption bands appeared in the range 230-200 nm (5.4-6.2 eV), like for 1,2-substituted MPC. 

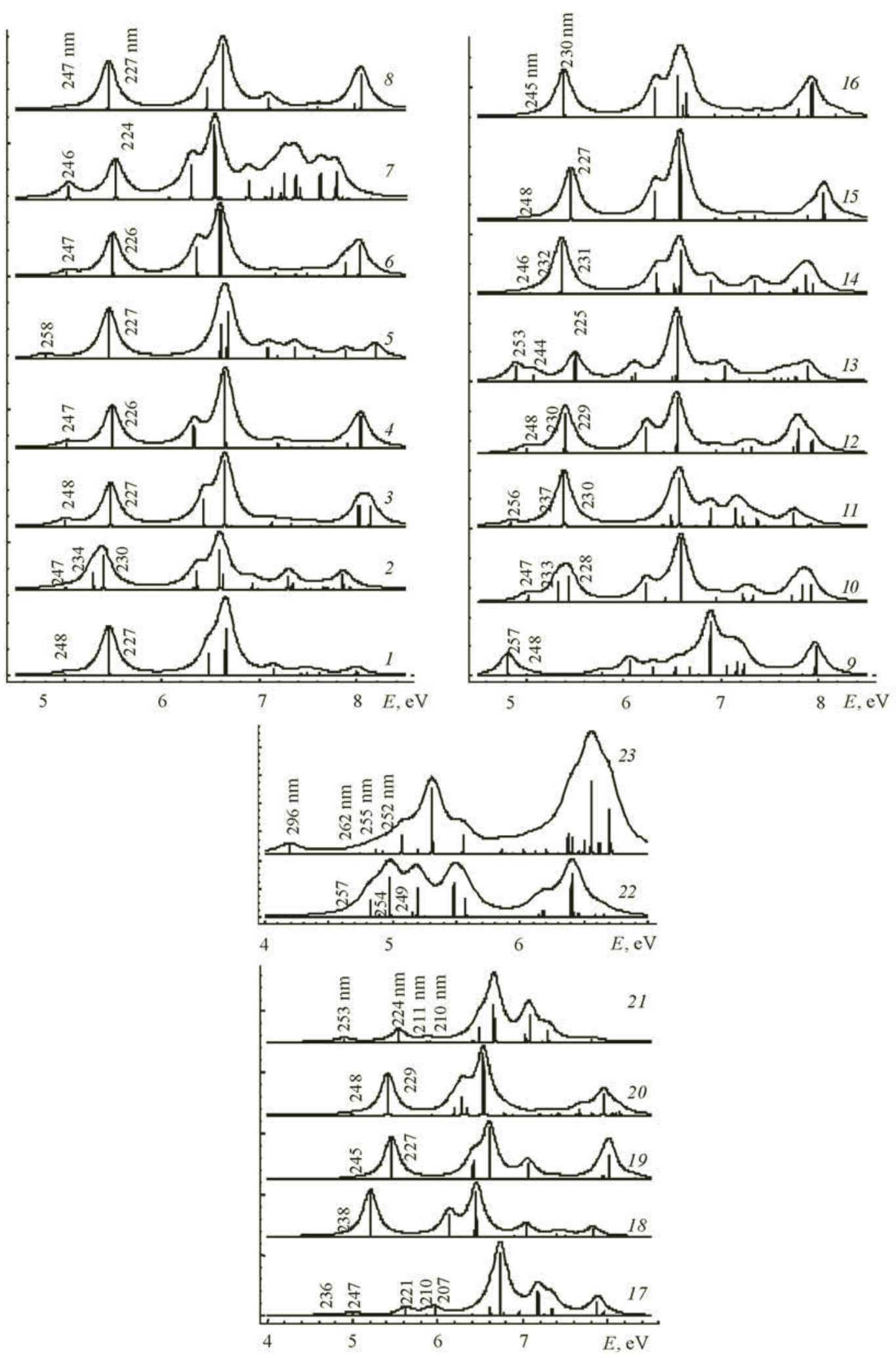

Fig. 2. Model electronic absorption spectra of methylphenylcarbamate (1): 1- (2); 2- (3); 3- (4); 4- (5); 5- (6); 6- (7); 7- (8); 1,2- (9); 1,3- (10); 1.4- (11); 1,5- (12); 1,6- (13); 1,7(14); 2,3- (15); 2,6- (16); 1,2,6- (equilibrium structure) (17); 1,2,6- (planar structure) (18); 7,8,9- (19); 2,3,5,6- (20); and 1,2,3,4,5,6-fluoro-substituted MPC (21); DPMC (22); and 1,2,6-fluoro-substituted DPMC (23). 
Fluorination of the methyl (7,8,9-substitution, spectrum 19) had practically no effect on the structure of the UV spectrum. The molecular structures (urethane group rotated by $21^{\circ}$ ) and spectra were practically identical for 2,3,5,6-substitution and 2,6-substitution (spectra 20 and 16).

In contrast with the gas phase, the mutual positioning of the urethane fragments could be different in the polymer structure as a result of local effects. Therefore, additional calculations with "frozen" coordinates for rotation of the urethane group relative to the ring plane with a value of zero and optimization of the geometry over all other coordinates were performed for 1,2,6-substituted MPC. As a result, a molecular configuration with an energy $55 \mathrm{~kJ} / \mathrm{mol}$ greater than the equilibrium value and that obviously corresponded to a transition state was obtained. The structures of spectra of the equilibrium (spectrum 17) and planar configuration (spectrum 18) of 1,2,6-substituted MPC also indicated that the conformational structures of urethane and its electronic absorption spectrum were related. The spectrum of the planar configuration of the 1,2,6-substituted derivative experienced less significant changes than MPC but noticeable ones. The $S_{1}$ and $S_{2}$ bands were shifted by 3 and $11 \mathrm{~nm}$ to longer wavelength.

Thus, all examined versions of F-substitution (with the exception of methyl fluorination) produced more or less significant changes in the molecular structure. In turn, this was reflected in the positions of the absorption bands and; therefore, could act as a spectral signature of fluorosubstitution. Fluorination of one of the DPMC fragments in the 1-, 2-, and 6-positions (Fig. 1c) also provided confirmation of the noted trends, i.e., rotation of the urethane group by $64^{\circ}$ was accompanied by characteristic restructuring of the UV absorption spectrum (spectra 22 and 23).

Conclusions. The electronic structures of fluorinated MPC and DPMC based on quantum-chemical TDDFT modeling were analyzed using changes of electronic absorption spectra due to different numbers and mutual positions of $\mathrm{F}$ atoms in the urethane structure. It was found that substitution of $\mathrm{H}$ atoms closest to the urethane generated steric hindrance in the urethane structure and rotation of the urethane group by a significant angle $\left(\sim 20^{\circ}\right)$. The rotation of the urethane group could reach $60^{\circ}$ and more upon additional fluorination of the NH group. Such a structural change of the urethane was accompanied by characteristic significant bathochromic shifts of the long-wavelength absorption bands. Fluorination of the ring meta-positions and the methyl had less significant effects on the shape of the electronic spectra. The established trends could be used to study the surface structure and composition of polymeric sorbing filters and to solve problems of polymer conformational analysis and correlation analysis of the sorption properties of polyurethane foams. This is being addressed in the Laboratory of the Physical Chemistry of Polymers and Natural Organic Compounds, A. N. Sevchenko IAPP, BSU.

Acknowledgments. We thank A. I. Stankevich for useful advice and recommendations.

\section{REFERENCES}

1. S. G. Dmitrienko and V. V. Apyari, Polyurethane Foams. Sorption Properties and Application in Chemical Analysis [in Russian], Krasand, Moscow (2009).

2. H.-G. Elias, Macromolecules, Vol. 4, Applications of Polymers, Wiley-VCH (2009).

3. G. V. Lisichkin (ed.), Chemistry of Grafted Surface Compounds [in Russian], Fizmatlit, Moscow (2003).

4. A. P. Kharitonov and B. A. Loginov, Ross. Khim. Zh., 52, No. 3, 106-111 (2008).

5. M. A. Ksenofontov, D. S. Umreiko, and M. B. Shundalov, Zh. Prikl. Spektrosk., 79, No. 3, 362-365 (2012).

6. K. Burke, J. Werschnik, and E. K. U. Gross, J. Chem. Phys., 123, 062206 (2005).

7. C. Adamo and V. Barone, Chem. Phys. Lett., 330, 152-160 (2000).

8. L. Miao, Y. Yao, F. Yang, Z. Wang, W. Li, and J. Hu, J. Mol. Struct.: THEOCHEM, 865, 79-87 (2008).

9. M. E. Casida, J. Mol. Struct.: THEOCHEM, 914, 3-18 (2009).

10. J. Fabian, Dyes Pigments, 84, 36-53 (2010).

11. M. Belletête, P.-L. T. Boudreault, M. Leclerc, and G. Durocher, J. Mol. Struct.: THEOCHEM, 962, 33-37 (2010).

12. M. W. Schmidt, K. K. Baldridge, J. A. Boatz, S. T. Elbert, M. S. Gordon, J. H. Jensen, S. Koseki, N. Matsunaga, K. A. Nguyen, S. J. Su, T. L. Windus, M. Dupuis, and J. A. Montgomery, J. Comput. Chem., 14, 1347-1363 (1993).

13. http://www.msg.ameslab.gov/GAMESS/GAMESS.html

14. B. M. Bode and M. S. Gordon, J. Mol. Graphics Modell., 16, 133-138 (1998).

15. L. J. Farrugia, J. Appl. Crystallogr., 30, 565 (1997).

16. Ya. F. Freimanis, Organic Compounds with Intramolecular Charge Transfer [in Russian], Zinatne, Riga (1985).

17. I. Iweibo, R. A. Oderinde, and J. A. Faniran, Spectrochim. Acta, Part A, 38, 1-7 (1982).

18. H. Iikura, T. Tsuneda, T. Yanai, and K. Hirao, J. Chem. Phys., 115, 3540-3544 (2001). 
19. O. Gritsenko and E. J. Baerends, J. Chem. Phys., 121, 655-660 (2004).

20. X.-H. Duan, X.-Y. Li, R.-X. He, and X.-M. Cheng, J. Chem. Phys., 122, 084314 (2005).

21. N. T. Maitra, J. Chem. Phys., 122, 234104 (2005).

22. W. Kohn, Rev. Mod. Phys., 71, 1253-1266 (1999).

23. T. Leininger, H. Stoll, H.-J. Werner, and A. Savin, Chem. Phys. Lett., 275, 151-160 (1997).

24. T. H. Dunning, Jr., J. Chem. Phys., 90, 1007-1023 (1989).

25. A. D. Becke, J. Chem. Phys., 98, 5648-5652 (1993).

26. C. Lee, W. Yang, and R. G. Parr, Phys. Rev. B: Condens. Matter Mater. Phys., 37, 785-789 (1988).

27. P. J. Stephens, F. J. Devlin, C. F. Chabalowski, and M. J. Frisch, J. Phys. Chem., 98, 11623-11627 (1994).

28. J. P. Doering, J. Chem. Phys., 67, 4065-4070 (1977).

29. V. Molina and M. Merchan, J. Phys. Chem. A, 105, 3745-3751 (2001).

30. T. Ebata, C. Minejima, and N. Mikami, J. Phys. Chem. A, 106, 11070-11074 (2002). 